\title{
Übersicht über die Gerontopsychiatrischen Zentren und Tageskliniken in Deutschland
}

In den letzten Jahren hat die Bedeutung der Gerontopsychiatrie und -psychotherapie nicht nur angesichts des demografischen Wandels erheblich zugenommen, sie erkämpft sich auch in der öffentlichen Wahrnehmung wachsende Aufmerksamkeit. Auch für die Gerontopsychiatrie ist „Ambulantisierung“ ein wichtiges versorgungspolitisches Stichwort. Von besonderer Bedeutung sind dabei die Gerontopsychiatrischen Zentren (GZ) mit Tagesklinik, Ambulanz und Beratung. Eine systematische Übersicht über ihre Anzahl und die regionale Verteilung wurde bisher für Deutschland nicht erstellt. Für die gerontopsychiatrischen Tageskliniken gab es allerdings von Wächtler [1], Hirsch et al. [2] und Wolter-Henseler [3] Übersichten. So war es an der Zeit, eine aktuelle Erhebung dieser Einrichtungen durchzuführen.

Um eine möglichst vollständige Übersicht über GZ und Tageskliniken zu erhalten, wurden alle Mitglieder der DGGPP (Deutsche Gesellschaft für Gerontopsychiatrie und -psychotherapie), des AK Gerontopsychiatrie der Bundesdirektorenkonferenz und einzelne Leiter von gerontopsychiatrischen Einrichtungen, deren Adressen nicht in den genannten Gruppen gelistet waren, angeschrieben. In einem vorgegebenen Fragebogen wurden die Daten für das Jahr 2018 im Folgejahr 2019 erhoben. Da es unterschiedliche Definitionen zum GZ gab (z.B. GZ als Überbegriff für stationäre und nichtstationäre Einrichtung, Alteneinrichtungen, die sich GZ nennen u.a.), wurde die von der Expertenkommission [4] beschriebene vorgegeben (Tagesklinik, Ambulanz, Altenberatung). Kann auch nicht von einer vollständigen Erhebung ausgegangen werden, so dürfte doch der größte Teil der GZ und TK bei dieser Befragung erfasst worden sein.

\section{Zur Ausgangslage}

In der Psychiatrie-Enquête [5] wurde für die nichtstationäre gerontopsychiatrische Versorgung empfohlen, für jedes Standardversorgungsgebiet (ca. 250000 Einwohner) ein Gerontopsychiatrisches Zentrum (GZ) einzurichten. Das GZ sollte die „zentrale Zusammenfassung dreier für die gerontopsychiatrische Versorgung besonders wichtige Dienste darstellen: Poliklinik, Tagesklinik und eine kleine stationäre Assessment-Unit“. Als Aufgabenschwerpunkte des GZ wurden neben der Patientenversorgung auch die Weiterbildung und Forschung explizit benannt. Ein besonderes Gewicht in der Versorgung wurde auf die Verbesserung der fachärztlichen Betreuung von Heimbewohnern gelegt. Auch wurde vorgeschlagen, in einigen Standardversorgungsgebieten einen Gerontopsychiatrischen Verbund unter Einbezug aller an der Versorgung Beteiligten zu erproben. Die Expertenkommission [4] stellte fest, dass die Versorgungssituation für psychisch kranke alte Menschen durch die nach der Psychiatrie-Enquête eingeleiteten Maßnahmen nahezu nicht beeinflusst worden sei. Sie empfahl keine stationäre „Assessment-Unit“ mehr, verwies vielmehr auf die zu verbessernde ambulante Diagnostik und auf gerontopsychiatrische Tageskliniken. Die Struktur der erneut vorgeschlagenen GZ, blieb dagegen unverändert. Die Bundesarbeitsgemeinschaft der Träger psychiatrischer Krankenhäuser stellte im Jahr 1997 [6] ein »Aktionsprogramm gerontopsychiatrische Versorgung" vor, in dem für jedes Versorgungsgebiet von 150000-250000 Einwohnern ein Gerontopsychiatrisches Zentrum ge- fordert und 30-50 klinische Betten/Plätze als angemessenes Angebot angesehen wurde, von denen $20-25 \%$ als teilstationär ausgewiesen sein sollten.

Entsprechend der Definition der Expertenkommission [4], die auch von den Fachgesellschaften getragen wird, spricht man von einem Gerontopsychiatrischen Zentrum (GZ), wenn in seinem Kernbestand eine teilstationäre Behandlungsund Rehabilitationseinrichtung (Tagesklinik), ein ambulanter Dienst und eine Altenberatung einbezogen sind oder - in der Entwicklungsphase - ein Baustein (Tagesklinik) verwirklicht ist und die weiteren in Kürze hinzukommen sollen. Die Aufgabe eines GZ sei, „treibende Kraft der gerontopsychiatrischen Versorgung“ (Expertenkommission 1988 [4]) in einer Region zu sein. Dazu zählt auch die aufsuchende Behandlung der Betroffenen und die Beratung der Angehörigen. Als zusätzliches Aufgabenfeld wurden die Öffentlichkeitsarbeit und die Aus-, Fortund Weiterbildung in der Versorgungsregion („Stimulator“) genannt. Das GZ sollte funktionell und räumlich eigenständig mit einem ärztlichen Leiter arbeiten. Die organisatorische Anbindung des GZ sollte je nach Gegebenheit einer Versorgungsregion erfolgen. Als Möglichkeiten wurden genannt:

- Anbindung an eine stationäre psychiatrische Versorgungseinrichtung,

- Anbindung an einen Träger, der für das Versorgungsgebiet maßgebende Einrichtungen der Altenhilfe betreibt,

- eigenständige Institution mit eigenständiger Trägerschaft.

Im Zwischenbericht der Enquête-Kommission „Demographischer Wandel“ [7] wurden die Empfehlungen der Expertenkommission erneut unterstrichen. Es wur- 
$\checkmark$ Tab. 1 Gerontopsychiatrische Zentren (GZ) (vorl. 015-03-2020 - Hirsch).

\begin{tabular}{|c|c|c|c|c|c|c|c|}
\hline Nr. & Ort & PLZ & Tageskl. & Ambul. & Altenber. & Mem/G. ${ }^{8}$ & Lehrkrh.-DAGPP \\
\hline 01 & Bernburg ${ }^{1}$ & 06406 & $x$ & $x$ & $x^{4}$ & & \\
\hline 02 & Berlin-Alexianer & 12526 & $x$ & $x$ & $x$ & $x$ & $x$ \\
\hline 03 & Güstrow & 18273 & $x$ & $x$ & $x$ & & $x$ \\
\hline 04 & Stralsund & 18437 & $x$ & $x$ & $x$ & $x$ & \\
\hline 05 & Schwerin & 19055 & $x$ & $x$ & $x$ & $x$ & \\
\hline 06 & Lüneburg & 21339 & $x$ & $x$ & $x$ & $x$ & \\
\hline 07 & Hamburg-Ochsenzoll & 22419 & $x$ & $x$ & $x^{4}$ & $x$ & $x$ \\
\hline 08 & Sehnde & 31319 & $X^{7}$ & $x$ & $x$ & $x$ & \\
\hline 09 & Detmold $^{5}$ & 32756 & $x$ & $x$ & $x$ & & \\
\hline 10 & Gütersloh & 33334 & $x$ & $x$ & $x$ & $x$ & \\
\hline 11 & Göttingen - Universität & 37075 & $\mathrm{x}$ & $x$ & $x^{4}$ & $x$ & \\
\hline 12 & Düsseldorf ${ }^{1,9}$ & 40629 & $x$ & $x$ & $x^{4}$ & $x$ & \\
\hline 13 & Langenfeld & 40764 & $x$ & $x$ & $x^{4}$ & $x$ & \\
\hline 14 & Viersen & 41749 & $x$ & $x$ & $x$ & $x$ & \\
\hline 15 & Solingen & 42653 & $x$ & $x$ & $x^{4}$ & $x$ & \\
\hline 16 & Dortmund & 44309 & $x$ & $x$ & $x$ & $x$ & \\
\hline 17 & Essen-LVR & 45147 & $x$ & $x$ & $x^{4}$ & $x$ & \\
\hline 18 & Krefeld & 47805 & $x$ & $x$ & $x$ & $x$ & $x$ \\
\hline 19 & Münster-LWL ${ }^{1}$ & 48147 & $x$ & $x$ & $x$ & $x$ & $x$ \\
\hline 20 & Münster-Alexianer & 48151 & $x$ & $x$ & $x$ & $x$ & \\
\hline 21 & Köln-Alexianer & 50996 & $x$ & $x$ & $x$ & & \\
\hline 22 & Köln-LVR & 51109 & $x$ & $x$ & $x$ & $x$ & $x$ \\
\hline 23 & Bonn-LVR & 53111 & $x$ & $x$ & $x$ & $x$ & $x$ \\
\hline 24 & Zülpich & 53901 & $x$ & $x$ & $x$ & & \\
\hline 25 & Alzey & 55232 & $x$ & $x$ & $x$ & $x$ & $x$ \\
\hline 26 & Iserlohn & 58644 & $x$ & $x$ & $x$ & $x$ & \\
\hline 27 & Berkamen & 59192 & $x$ & $x$ & $x$ & & \\
\hline 28 & Mannheim & 68159 & $x$ & $x$ & $x$ & $x$ & \\
\hline 29 & Wiesloch & 69168 & $x$ & $x$ & $x$ & & \\
\hline 30 & Stuttgart ${ }^{10}$ & 70374 & $x$ & $x$ & $x$ & $x$ & $x$ \\
\hline 31 & Reutlingen & 72764 & $x$ & $x$ & $x$ & $x$ & \\
\hline 32 & Weinsberg $^{3}$ & 74189 & $x$ & $x$ & $x$ & $x$ & $x$ \\
\hline 33 & Klingenmünster & 76889 & $x$ & $x$ & $x$ & & \\
\hline 34 & München Uni & 80336 & $x$ & $x$ & $x^{4}$ & $x$ & \\
\hline 35 & München TU & 81675 & $x$ & $x$ & $x$ & $x$ & \\
\hline 36 & Kaufbeuren & 87600 & $x$ & $x$ & $x$ & & \\
\hline 37 & Memmingen & 87700 & $X^{7}$ & $x$ & $x^{4}$ & $x$ & \\
\hline 38 & Ravensburg & 88214 & $x$ & $x$ & $x^{4}$ & $x$ & $x$ \\
\hline 39 & München-Haar & 85540 & $x$ & $x$ & $x$ & & $x$ \\
\hline 40 & Bayreuth & 95445 & $x$ & $x$ & $x$ & $x$ & $x$ \\
\hline
\end{tabular}


$>$ Tab. 1 (Fortsetzung)

\begin{tabular}{|c|c|c|c|c|c|c|c|}
\hline Nr. & Ort & PLZ & Tageskl. & Ambul. & Altenber. & Mem/G. ${ }^{8}$ & Lehrkrh.-DAGPP \\
\hline \multicolumn{8}{|c|}{ Hat die Bausteine Tagesklinik (TK) und gerontopsychiatrische Ambulanz, keine Altenberatung (nennt sich GZ) } \\
\hline 01 & Bielefeld ${ }^{2}$ & 33615 & $\mathrm{x}$ & $\mathrm{x}$ & nein & $x$ & $\mathrm{x}$ \\
\hline 02 & Bedburg-Hau & 47551 & $x$ & $x$ & nein & $x$ & \\
\hline 03 & Bonn - Universität ${ }^{2}$ & 53123 & $x$ & $x$ & nein & $x$ & \\
\hline 04 & Kempten ${ }^{2}$ & 87435 & $x$ & $x$ & nein & $x$ & \\
\hline \multicolumn{8}{|c|}{ Hat die Bausteine TK u. gerontopsych. Amb., keine Altenberatung (nennt sich nicht GZ) } \\
\hline 01 & Altenburg & 04600 & $x$ & $x$ & nein & $x$ & \\
\hline 02 & Berlin-Spandau & 13585 & $x$ & $x$ & $X^{4}$ & nein & \\
\hline 03 & Greifswald & 17489 & $x$ & $x$ & nein & $x$ & \\
\hline 04 & Liebenburg & 38704 & $x$ & $x$ & nein & $x$ & \\
\hline 05 & Duisburg & 47053 & $x$ & $x$ & nein & nein & \\
\hline 06 & Saarbrücken & 66119 & $x$ & $x$ & $x^{4}$ & $x$ & $x$ \\
\hline 07 & Winnenden & 71364 & $x$ & $x$ & nein & nein & \\
\hline 08 & Landsberg am Lech & 86899 & $x$ & $x$ & nein & nein & \\
\hline 09 & Nürnberg & 90419 & $x$ & $x$ & nein & $x$ & $x$ \\
\hline 10 & Ansbach & 91522 & $x$ & $x$ & nein & $x$ & \\
\hline
\end{tabular}

Hat die Bausteine gerontopsych. Ambulanz u. Altenberatung; keine Tagesklinik (nennt sich nicht GZ)

\begin{tabular}{|l|l|l|l|l|l|l|l|}
\hline 01 & Bad Zwischenahn & 26160 & nein & $X$ & $X$ & nein & \\
\hline 02 & Reichenau & & & \\
\hline 03 & Erlangen & 78479 & nein & $X$ & $X$ & $X$ & $X$ \\
\hline
\end{tabular}

Hat eine integrierte TK (stationär/Allgemeinpsychiatrie) und gerontopsych. Ambulanz; keine Altenberatung (nennt sich nicht GZ)

\begin{tabular}{|l|l|l|l|l|l|}
\hline 01 & Bernburg & 06406 & $X^{7}$ & nein & nein \\
\hline 02 & Rüdersdorf b. Berlin & 15562 & $X^{6}$ & $X$ & nein \\
\hline 03 & Haina & 35114 & $X^{6}$ & $X$ & $X$ \\
\hline 04 & Lengerich & 49525 & $X^{6,7}$ & $X$ & $X^{4}$ \\
\hline 05 & Andernach & 56626 & $X^{7}$ & $X$ & nein \\
\hline
\end{tabular}

${ }^{1}$ hat alle Bausteine des GZs: nennt sich offiziell nicht so

${ }^{2}$ hat TK u. gerontopsych. Amb., keine Altenberatung/nennt sich GZ

${ }^{3}$ hat TK u. gerontopsych. Amb., keine Altenberatung/nennt sich nicht GZ

${ }^{4}$ hat GZ: Altenberatung in Zusammenarbeit mit örtl. Beratungsstellen

${ }^{5}$ hat gerontopsych. Amb. und Altenberatung; keine TK

${ }^{6}$ integrierte TK in stationäre $u$. allgemeinpsychiatrische TK

${ }^{7}$ integrierte TK in stationäre Gerontopsychiatrie

${ }^{8}$ Mem/G.: MemoryClinic/Gedächtnissprechstunde

${ }^{9}$ Entwicklung zum Zentrum für Altersmedizin

${ }^{10}$ Zentrum für Altersmedizin

11 hat keine TK, nennt sich GZ

de darauf hingewiesen, dass das GZ gerontopsychiatrischen Abteilungen oder psychiatrischen Abteilungen am Allgemeinkrankenhaus angegliedert werden kann. Wie sich gezeigt hat, sind fast alle $G Z$, die derzeit bestehen, mit einer gerontopsychiatrischen Abteilung eines Landes-/Bezirkskrankenhauses oder einer Klinik verbunden.
Inzwischen kann die Effizienz der Arbeitsweise eines GZ schon lange als nachgewiesen gelten [8]. Wie sich gezeigt hat, tragen die derzeit bestehenden GZ und Einrichtungen, die in ähnlicher Weise arbeiten, zu einer erheblichen Verbesserung der Versorgung, der Zusammenarbeit der einzelnen Einrichtungen und einem regionalen, vermehrten und diffe- renzierteren, öffentlichen Problembewusstsein bei [9]. Auch wenn die Angaben über die Zahl der notwendigen Versorgungsangebote im Prozess der Psychiatriereform manche Änderung erfuhren, wurde von sozialpolitischen Experten trotz geringfügiger Differenzen in der Einschätzung der zur Versorgung notwendigen Quantitäten - jüngere Quellen 
Tab.2 Übersicht über die Tageskliniken.

\begin{tabular}{|c|c|c|c|c|c|c|c|c|c|}
\hline Nr. & Ort & PLZ & $\begin{array}{l}\text { Geronto } \\
\text { TK }\end{array}$ & $\begin{array}{l}\text { geronto/ } \\
\text { allg- } \\
\text { Psych }^{2}\end{array}$ & $\begin{array}{l}\text { geronto/ } \\
\text { TK ger- } \\
\text { iatr. }^{3}\end{array}$ & $\begin{array}{l}\text { geronto/ } \\
\text { neurol. }{ }^{4}\end{array}$ & $\begin{array}{l}\text { geronto/ } \\
\text { station. }^{6}\end{array}$ & Plätze & $\begin{array}{l}\text { Anzahl } \\
\text { Beh. } \\
2018^{5}\end{array}$ \\
\hline
\end{tabular}

gerontopsychiatrische Tageskliniken

\begin{tabular}{|c|c|c|c|c|c|c|c|}
\hline 01 & Altenburg & 04600 & $x$ & & & 9 & k. A. ${ }^{a}$ \\
\hline 02 & Großschweidnitz & 02708 & $x$ & & & 15 & k. A. \\
\hline 03 & Berlin-Spandau & 13585 & $x$ & & & 12 & 123 \\
\hline 04 & Berlin-Hedwigshöhe & 12526 & $x$ & & & 15 & 178 \\
\hline 05 & Greifswald & 17489 & $x$ & & & 10 & 98 \\
\hline 06 & Güstrow & 18273 & $x$ & & & 12 & 64 \\
\hline 07 & Stralsund & 18437 & $x$ & & & 10 & 98 \\
\hline 08 & Schwerin & 19055 & $x$ & & & 20 & k. A. \\
\hline 09 & Lüneburg & 21339 & $x$ & & & 10 & 86 \\
\hline 10 & Hamburg-Ochsenzoll & 22419 & $x$ & & & k. A. & k. A. \\
\hline 11 & Detmold & 32756 & $x$ & & & $20-22$ & k. A. \\
\hline 12 & Paderborn & 33098 & $x$ & & & 12,5 & 98,5 \\
\hline 13 & Gütersloh & 33334 & $x$ & $x$ & $x$ & $24 / 6^{6}$ & 198 \\
\hline 14 & Bielefeld & 33615 & $x$ & & & 16 & 142 \\
\hline 15 & Göttingen-Universität & 37075 & $x$ & & & 25 & 200 \\
\hline 16 & Liebenburg & 38704 & $x$ & & & 15 & k. A. \\
\hline 17 & Düsseldorf & 40629 & $x$ & & & 25 & 156 \\
\hline 18 & Langenfeld & 40764 & $x$ & & & 17 & k. A. \\
\hline 19 & Viersen & 41749 & $x$ & & & 16 & 99 \\
\hline 20 & Solingen & 42653 & $x$ & & & 17 & k. A. \\
\hline 21 & Dortmund & 44309 & $x$ & & & 20 & 135 \\
\hline 22 & Essen-LVR & 45147 & $x$ & & & k. A. & k. A. \\
\hline 23 & Duisburg & 47053 & $x$ & & & 16 & k. A. \\
\hline 24 & Bedburg-Hau & 47551 & $x$ & & & 15 & 112,5 \\
\hline 25 & Krefeld & 47805 & $x$ & & & 12 & 100 \\
\hline 26 & Münster-LWL & 48147 & $x$ & & & 11 & 91 \\
\hline 27 & Münster-Alexianer & 48151 & $x$ & & & k. A. & k. A. \\
\hline 28 & Köln-Alexianer & 50996 & $x$ & & & 15 & 87 \\
\hline 29 & Köln-LVR & 51109 & $x$ & & & 2-mal 18 & k. A. \\
\hline 30 & Bonn-LVR & 53111 & $x$ & & & 18 & 131 \\
\hline 31 & Bonn-Universität & 53123 & $x$ & & & $15(+3)$ & 98 \\
\hline 32 & Zülpich/Hürth & 53909 & $x$ & $x$ & & $13 / 34$ & 460 \\
\hline 33 & Iserlohn & 58644 & $x$ & & & 18 & k. A. \\
\hline 34 & Berkamen & 59192 & $x$ & & & 20 & 117 \\
\hline 35 & Saarbrücken & 66119 & $x$ & & & 15 & 108 \\
\hline 36 & Mannheim & 68159 & $x$ & & & 12 & 150 \\
\hline 37 & Wiesloch & 69168 & $x$ & & & 16 & 131 \\
\hline
\end{tabular}


- Tab.2 (Fortsetzung)

\begin{tabular}{|c|c|c|c|c|c|c|c|c|c|}
\hline Nr. & Ort & PLZ & $\begin{array}{l}\text { Geronto } \\
\text { TK }\end{array}$ & $\begin{array}{l}\text { geronto/ } \\
\text { allg- } \\
\text { Psych }^{2}\end{array}$ & $\begin{array}{l}\text { geronto/ } \\
\text { TK ger- } \\
\text { iatr. }^{3}\end{array}$ & $\begin{array}{l}\text { geronto/ } \\
\text { neurol. }{ }^{4}\end{array}$ & $\begin{array}{l}\text { geronto/ } \\
\text { station. }^{6}\end{array}$ & Plätze & $\begin{array}{l}\text { Anzahl } \\
\text { Beh. } \\
2018^{5}\end{array}$ \\
\hline 38 & Stuttgart & 70374 & $x$ & & & & & 24 & $148^{\mathrm{b}}$ \\
\hline 39 & Winnenden & 71364 & $x$ & & & & & 19 & 88 \\
\hline 40 & Reutlingen & 72764 & $x$ & & & & & 16 & k. A. \\
\hline 41 & Weinsberg & 74189 & $x$ & & & & & 21 & 166 \\
\hline 42 & Klingenmünster & 76889 & $x$ & & & & & $6^{6}$ & 3 \\
\hline 43 & München-TU & 81675 & $x$ & & & & & 12 & 250 \\
\hline 44 & Landsberg am Lech & 86899 & $x$ & & & & & 15 & 90 \\
\hline 45 & Kempten & 87435 & $x$ & & & & & 10 & k. A. \\
\hline 46 & Kaufbeuren ${ }^{6}$ & 87600 & $x$ & & & & & k. A. & $5-10$ \\
\hline 47 & Ravensburg & 88214 & $x$ & & & & & k. A. & k. A. \\
\hline 48 & München-Haar & 85540 & $x$ & & & & & 18 & $\begin{array}{l}\text { Mitte } \\
2018 \\
\text { eröffnet }\end{array}$ \\
\hline 49 & Bayreuth & 95445 & $x$ & & & & & 6 & 20 \\
\hline \multicolumn{10}{|c|}{ „gemischte“ Tageskliniken } \\
\hline 01 & Bernburg & 06406 & & & & & $x$ & 5 & 28 \\
\hline 02 & Rüdersdorf bei Berlin & 15562 & & $x$ & & & & k. A. & k. A. \\
\hline 03 & Sehnde ${ }^{1}$ & 31319 & & $x$ & & & & 15 & k. A. \\
\hline 04 & Haina & 35114 & & $x$ & & & $x$ & $3^{2} / 3^{6}$ & 15 \\
\hline 05 & Göttingen-Asklepios & 37081 & & & $x$ & & & 21 & 144 \\
\hline 06 & Lengerich & 49525 & & $x$ & & & $x$ & 2 & k. A. \\
\hline 07 & Alzey & 55232 & & $x$ & & & & 4 & 40 \\
\hline 08 & Bad Kreuznach & 55583 & & $x$ & $x$ & & & 15 & $\begin{array}{l}\text { Dez. } 2019 \\
\text { eröffnet }\end{array}$ \\
\hline 09 & Andernach & 56626 & & & $x$ & & $x$ & $3-6$ & 20 \\
\hline 10 & Hochschweizen & 64720 & & & $x$ & & & $3-6$ & 20 \\
\hline 11 & München Uni & 80336 & & & $x$ & $x$ & & $5 / 5$ & k. A. \\
\hline 12 & Memmingen & 87700 & & $x$ & & & & 12 & 129 \\
\hline 13 & Nürnberg & 90419 & & & $x$ & & & 37 & k. A. \\
\hline 14 & Ansbach & 91522 & & & & & $x$ & 6 & 45 \\
\hline 15 & Deggendorf-Mainkofen & 94469 & & & & & $x$ & k. A. & k. A. \\
\hline \multicolumn{10}{|c|}{$\begin{array}{l}1 \text { integrierte TK in stationär u. allgemeinpsychiatrische TK } \\
{ }^{2} \text { gemischte geronto-/allgemeinpsychiatrische Tagesklinik } \\
3 \text { gemischte geronto-/geriatrische Tagesklinik } \\
{ }^{4} \text { gemischte geronto-/neurologische Tagesklinik } \\
{ }^{5} \text { Anzahl der Behandlungen im Jahr } 2018 \\
{ }^{6} \text { stationsintegrierte gerontopsychiatrische Tagesklinik } \\
\text { a Beginn 2018, } 2019 \text { regelhafte Behandlungen } \\
\text { b zuzüglich } 4 \text { Plätze in stationsbezogener TK in Bad Cannstatt }\end{array}$} \\
\hline
\end{tabular}


tendieren zu etwas niedrigeren Werten mehrfach einhellig die Notwendigkeit eines spezifischen gerontopsychiatrischen Versorgungsangebots festgestellt [10]. Allen diesen Vorschlägen ist das Ziel gemeinsam, in Deutschland Versorgungsbedingungen zu schaffen, wie sie in anderen europäischen Ländern (z.B. Großbritannien) schon zu Zeiten der Expertenkommission bestanden und seitdem ständig weiterentwickelt wurden.

\section{Übersicht über die GZs}

Nach einiger Verzögerung wurden seit 1991 GZ eingerichtet. Die erste Gründung eines GZ geschah in Gütersloh 1991. Es folgten bis 1998 noch 7 weitere (Bonn, Eberswalde, Kaufbeuren, Kempten, Leipzig, Münster, Osnabrück) sowie einige Einrichtungen, die Bausteine des GZ aufwiesen und sich zu GZ weiterentwickeln wollten [11].

Aus der aktuellen Erhebung geht hervor, dass über ganz Deutschland verteilt 40 GZ bestehen, von denen 13 als Lehrkrankenhaus der DAGPP (Deutsche Akademie für Gerontopsychiatrie und -psychotherapie) anerkannt sind ( $\triangleright$ Tab. 1 ). Daneben existieren 22 Einrichtungen, die unterschiedliche Bausteine eines GZ aufweisen und meist auch planen, ein vollständiges GZ zu schaffen. Von ihnen sind 4 auch Lehrkrankenhäuser der DAGPP. Der überwiegende Teil der GZ hat auch eine Memoryklinik (30 GZ und 12 der Einrichtungen mit Anteilen eines $G Z$ ). Eine Liste aller Memorykliniken in Deutschland wurde von der Hirnliga (http://www.hirnliga.de/ frueh-erkennung) erstellt. Die Altenberatung üben einige $G Z$ in Zusammenarbeit mit örtlichen Beratungsstellen aus.

\section{Übersicht über die Tageskliniken}

Die Entwicklung von gerontopsychiatrischen Tageskliniken (TK) begann in Deutschland sehr zögerlich. Die erste Tagesklinik wurde in Hamburg 1976 eingerichtet. 15 Jahre später existieren 15 TK [1]. 1998 gab es 29 gerontopsychiatrische TK (die meisten in Nordrhein-Westfalen) zuzüglich 11 "gemischte“ Einrichtungen [2]. 2001 gab es schon 37 gerontopsychiatrische TK (Wächtler 2004). In der aktuellen Untersuchung konnten für das Jahr 2018 insgesamt 49 spezialisierte und 15 "gemischte“ TK identifiziert werden (\Tab.2). Die Anzahl der Behandlungsplätze lag zwischen 6 und 34 (Durchschnitt: 16), die der "gemischten“ TK zwischen 2 und 37 (Durchschnitt: 11).

\section{Zusammenfassung und Ausblick}

Betrachtet man die Forderungen der Psychiatrie-Enquête (1975) und der Experten-Kommission (1988), so sind diese, trotz der positiven Erfahrungen mit den bestehenden GZ, bei Weitem nicht erfüllt worden. Dies erstaunt umso mehr, da der Anteil älterer Menschen - und damit auch der psychisch Kranken - in unserer Gesellschaft stetig steigt. Zur Diskussion steht, ob eine Entwicklung zum „Zentrum für Altersmedizin“ bzw. „Zentrum für Ältere“ (Wächtler et al. [12, 13]) in Kooperation mit der Geriatrie in Zukunft versorgungsgünstiger ist und ob solche kombinierten Strukturen eine größere Realisierungschance bei Trägern von Einrichtungen und der Politik haben. Auch von einer angemessenen Versorgung durch gerontopsychiatrische Tageskliniken kann man noch nicht sprechen, obwohl ihre Position in der regionalen gerontopsychiatrischen Versorgungsstruktur gut begründet ist und sie sich vielfach als unverzichtbare Bausteine erwiesen haben. Weder ist ihre Zahl auch nach konservativsten Planungsvorgaben ausreichend (weit über $350 \mathrm{GZ}$ inklusive TK wären erforderlich), noch ist der angestrebte Wert für tagesklinische Behandlungsplätze von 20-25\% der gerontopsychiatrischen stationären Plätze auch nur annähernd erreicht, wie es für die Allgemeinpsychiatrie bundesweit in Näherung bereits Standard ist.

Diverse Modellprogramme, Modellregionen, Demenznetze, Pflegestützpunkte und schließlich auch das "Leuchtturmprojekt Demenz« sowie das Deutsche Zentrum für Neurodegenerative Erkrankungen (DZNE) der Bundesregierung sind zu begrüßende Initiativen. Das Gerontopsychiatrische Zentrum im Versorgungsverbund als verpflichtendes Kernelement der gerontopsychiatrischen Regelversorgung harrt aber in weiten Teilen noch seiner Umsetzung. Nach dem Konsensus-
Statement der Weltgesundheitsorganisation und des Weltverbands der Psychiater [14] ist eine kompetente Gerontopsychiatrie und -psychotherapie unverzichtbar und nicht durch andere Fachgebiete, etwa die Geriatrie, oder die allgemeinpsychiatrische Kompetenz zu ersetzen. Für eine angemessene Versorgung einer Region ist es nicht akzeptabel, nur einen altersübergreifenden allgemeinpsychiatrischen Dienst anzubieten, wie es an vielen Stellen in unserem Land weiterhin Standard ist. Mit dem Royal College of Psychiatrists muss ein solches Vorgehen ohne Einschränkung als Altersdiskriminierung bezeichnet werden [15].

Sehr herzlich möchten wir uns bei allen Teilnehmerinnen und Teilnehmern der Befragung bedanken. Ohne sie wäre diese Übersicht nicht möglich gewesen.

Rolf. D. Hirsch, Hans Gutzmann, Sebastian Schwandt, Bonn/Berlin

Literatur

[1] Wächtler C. Die Gerontopsychiatrische Tagesklinik. Regensburg: Roderer; 1997

[2] Hirsch RD, Holler G, Reichwald W, Gervink T. Leitfaden für die ambulante und teilstationäre gerontopsychiatrische Versorgung. Schriftenreihe des BMG, Band 114. BadenBaden: Nomos; 1999

[3] Wolter-Henseler DK. Gerontopsychiatrische Tageskliniken. In: Eikelmann B, Reker T, Hrsg. Psychiatrie und Psychotherapie in der Tagesklinik. Stuttgart: Kohlhammer; 2004: 83-93

[4] Bundesministerium für Jugend, Familie, Frauen und Gesundheit. Empfehlungen der Expertenkommission der Bundesregierung zur Reform der Versorgung im psychiatrischen und psychotherapeutisch/psychosomatischen Bereich. Bonn: 1988

[5] Deutscher Bundestag. Bericht über die Lage der Psychiatrie in der Bundesrepublik Deutschland - Zur psychiatrischen und psychotherapeutisch/psychosomatischen Versorgung der Bevölkerung. Drucksache 7/ 4200 u. 7/4202. Bonn: Heger; 1975

[6] Bundesarbeitsgemeinschaft der Träger Psychiatrischer Krankenhäuser (BAG Psychiatrie). Bericht über den Stand der klinischgerontopsychiatrischen Versorgung in der Bundesrepublik Deutschland. Bonn: 1997

[7] Deutscher Bundestag. Zwischenbericht der Enquete-Kommission „Demographischer Wandel“. Herausforderung unserer älter werdenden Gesellschaft an den Einzelnen 
und die Politik. Drucksache 12/876. Bonn: 1994

[8] Steinkamp G, Werner B. Effekte eines Gerontopsychiatrischen Zentrums auf das regionale Versorgungssystem psychisch gestörter älterer Menschen. Opladen: Leske + Budrich; 1997

[9] Remlein K-H, Netz P. Von der Siechenstation zum Gerontopsychiatrischen Zentrum. Gütersloh: van Hoddis; 1996

[10] Gutzmann H, Klein U. Gemeindebezogene gerontopsychiatrische Intervention. In: Wahl H-W, Tesch-Römer C, Ziegelmann JP, Hrsg. Angewandte Gerontologie. Stuttgart: Kohlhammer; 2012: 535-540

[11] Hirsch RD. Das gerontopsychiatrische Zentrum - (k)eine Zukunft? In: Remlein K-H, Netz P, Hrsg. Von der Siechenstation zum
Gerontopsychiatrischen Zentrum. Gütersloh: van Hoddis; 1996: 86-110

[12] Wächtler C, Clavijo J, Hofmann W. Das Zentrum für Ältere - Kooperation von Geriatrie und Gerontopsychiatrie bei der Versorgung psychisch und körperlich kranker alter Menschen. In: Möller H-J et al., Hrsg. Gerontopsychiatrie als interdisziplinäre Aufgabe. Stuttgart: Kohlhammer; 2004: 201 - 208

[13] Wächtler C. Die gerontopsychiatrischen Tageskliniken - 25 Jahre Erfahrung in der Bundesrepublik. In: Wächtler C, Jacob U, Helmig S, Hrsg. Die gerontopsychiatrischen Tageskliniken in der Bundesrepublik Deutschland. Hamburg: Eigendruck Klinikum Nord; 2004: 12 - 19

[14] World Health Organization \& World Psychiatric Association (WHO \& WPA). Orga- nization of care in psychiatry of the elderly a technical consensus statement. Aging Mental Health 1998; 2: 246-252

[15] Royal College of Psychiatrists. Age discrimination in mental health services: making equality a reality. 2009: http://rcpsych.ac. uk/pdf/PS02_2009x.pdf (Zugriff am 18.06.2020)

\section{Korrespondenzadresse}

Prof. Dr. Dr. Rolf D. Hirsch Bornheim

Deutschland

r.d.hirsch@t-online.de 